\title{
NONLINEAR ELLIPTIC EQUATIONS ON THE UPPER HALF SPACE
}

\author{
SUFANG TANG, LEI WANG AND MEIJUN ZHU
}

\begin{abstract}
In this paper we shall classify all positive solutions of $\Delta u=a u^{p}$ on the upper half space $H=\mathbb{R}_{+}^{n}$ with nonlinear boundary condition $\partial u / \partial t=-b u^{q}$ on $\partial H$ for both positive parameters $a, b>0$. We will prove that for $p \geq$ $(n+2) /(n-2), 1 \leq q<n /(n-2)$ (and $n \geq 3)$ all positive solutions are functions of last variable; for $p=(n+2) /(n-2), q=n /(n-2)$ (and $n \geq 3$ ) positive solutions must be either some functions depending only on last variable, or radially symmetric functions.
\end{abstract}

Keywords Curvature equation, Kelvin transform, Moving plane method, Moving sphere method

\section{Introduction}

Let $H=\mathbb{R}_{+}^{n}=\left\{\left(x^{\prime}, t\right) \mid x^{\prime}=\left(x_{1}, x_{2}, \ldots, x_{n-1}\right) \in \mathbb{R}^{n-1}, t>0\right\}$ be the upper half space in $\mathbb{R}^{n}$ with $n \geq 3$. We are interested in the following elliptic equation with a nonlinear boundary condition.

$$
\left\{\begin{array}{l}
\Delta u=a u^{p}, \quad u \geq 0 \quad \text { in } \quad H=\mathbb{R}_{+}^{n}, \\
\frac{\partial u}{\partial t}=b u^{q} \quad \text { on } \quad \partial H=\mathbb{R}^{n-1} .
\end{array}\right.
$$

Equation (1.1) was early studied by J. Escobar 2 in his work on Yamabe problem on compact manifolds with boundary. Let $\left(M, g_{0}\right)$ be a compact Riemannian manifold with boudary of dimension $n \geq 3$. The Yamabe problem is to find metrics $g$ conformally equivalent to $g_{0}$ of constant scalar curvature $R_{g}$, with constant mean curvature $h_{g}$ on $\partial M$. When $M$ is the unit ball in $\mathbb{R}^{n}$, since $M$ is conformall equivalent to $\mathbb{R}_{+}^{n}$, the problem is reduced to equation (1.1) for the critical case $\left(p=\frac{n+2}{n-2}\right.$ and $q=\frac{n}{n-2}$ ). With suitable decay assumption, Escobar was able to obtain the classification result for $a<0, b<0, p=\frac{n+2}{n-2}$ and $q=\frac{n}{n-2}$ (which mean $R_{g} \geq 0$ and $h_{g}<0$ for the manifold). Later, the decay condition was removed by Li and Zhu [7, and many interesting results on the classification of positive solutions of equation (1.1) for general cases appeared. We summarize the known results below.

(i) For $a=0, b<0, \mathrm{Hu}[5]$ showed that there is no positive classical solution for subcritical case $\left(1 \leq q<\frac{n}{n-2}\right.$ and $\left.n \geq 3\right)$; For critical case $q=\frac{n}{n-2}$, Ou [9] proved that all positive classical solutions are fundamental solutions of the Laplace equation multiplied by proper constants. The classical moving plane method (see, for example, [4]) was used in these papers.

(ii) For $a<0, b \in \mathbb{R}$, by introducing the method of moving spheres, Li and Zhu [7. classified all nonnegative classical solutions without the decay assumption at 
infinity for the critical case $p=\frac{n+2}{n-2}, q=\frac{n}{n-2}$ (and $n \geq 3$ ). See, also the paper by Chipot, Shafrir and Fila [1]

(iii) For $a \geq 0, b>0$, Lou and Zhu [ 8 classified all nonnegative classical solutions for $q>1$ and $n \geq 2$ if $a=0, b>0$; and for $p, q>1$ and $n \geq 2$ if $a, b>0$ by using the method of moving planes and the technics of lifting dimensions.

(iv) For $a<0, b<0, \mathrm{Li}$ and Zhang [6] proved that there is no positive classical solution for $0 \leq p \leq \frac{n+2}{n-2},-\infty<q \leq \frac{n}{n-2}, p+q<\left(\frac{n+2}{n-2}\right)+\left(\frac{n}{n-2}\right)$ (and $n \geq 3$ ) by using the method of moving spheres.

In this paper, we shall study the remaining case: $a>0, b<0$. After rescaling, it suffices to consider the following problem.

$$
\left\{\begin{array}{l}
\Delta u=n(n-2) u^{p}, \quad u>0 \text { in } H, \\
\frac{\partial u}{\partial t}=-c_{+} u^{q} \quad \text { on } \quad \partial H
\end{array}\right.
$$

for a positive constant $c_{+}$.

We first point out the specialty of this case: there might be more than two types of positive solutions. In fact, one can check that for $x=\left(x^{\prime}, t\right) \in H$ and $n \geq 3$, both

$$
u\left(x^{\prime}, t\right)=\left[x^{2}+(d+t)^{2}\right]^{-\gamma} \text { and } u\left(x^{\prime}, t\right)=\left(d A^{-1}+A t\right)^{-2 \gamma}
$$

satisfy the following elliptic equation:

$$
\left\{\begin{array}{l}
\Delta u=2 \gamma(2 \gamma+2-n) u^{p}, \quad u>0 \text { in } H, \\
\frac{\partial u}{\partial t}=-2 d \gamma u^{p} \quad \text { on } \quad \partial H,
\end{array}\right.
$$

where $d>0, \gamma>\frac{n-2}{2}, A=[(2 \gamma+2-n) /(2 \gamma+1)]^{1 / 2}$ and $p=1+\frac{1}{\gamma}$, which implies $p<\frac{n}{n-2}$.

Our first result can be stated as follows.

Theorem 1.1. If $u(x) \in C^{2}(H) \cap C^{1}(\bar{H})$ solves (1.2) and $n \geq 3, p \geq \frac{n+2}{n-2}, 1 \leq$ $q<\frac{n}{n-2}$, then $u=\left(\frac{p-1}{2} A t+B\right)^{-2 /(p-1)}$, where $A=\left(\frac{2 n(n-2)}{p+1}\right)^{1 / 2}$ and $B=$ $\left(c_{+}^{-1} A\right)^{-(p-1) /(2 q-p-1)}$.

Apparently, the supercritical power assumption (that is $p \geq \frac{n+2}{n-2}$ ) is needed in Theorem 1.1 due to the above mentioned example.

For critical case, we have

Theorem 1.2. If $u(x) \in C^{2}(H) \cap C^{1}(\bar{H})$ solves (1.2) and $n \geq 3, p=\frac{n+2}{n-2}, q=\frac{n}{n-2}$. Then for $c_{+} \neq n-2, u\left(x^{\prime}, t\right)=\left(\frac{\varepsilon}{\left|\left(x^{\prime}, t\right)-\left(x_{0}^{\prime}, t_{0}\right)\right|^{2}-\varepsilon^{2}}\right)^{(n-2) / 2}$; for $c_{+}=n-2$, either $u\left(x^{\prime}, t\right)=\left(\frac{\varepsilon}{\left|\left(x^{\prime}, t\right)-\left(x_{0}^{\prime}, t_{0}\right)\right|^{2}-\varepsilon^{2}}\right)^{(n-2) / 2}$ or $u\left(x^{\prime}, t\right)=u(0, t)=\left(2 t+u(0)^{-2 /(n-2)}\right)^{-(n-2) / 2}$, where $0<\varepsilon<\left((n-2)^{-1} c_{+}\right)^{2 / n}, x_{0}^{\prime} \in \mathbb{R}^{n-1}$, and $t_{0}=-c_{+}(n-2)^{-1} \varepsilon^{-(n-2) / 2}$.

If both $p>\frac{n+2}{n-2}$ and $q>\frac{n}{n-2}$, solutions of (1.2) are complicated. We have the following observation.

(1) If $2 q \neq p+1$, then $u=\left(\frac{p-1}{2} A t+B\right)^{-2 /(p-1)}$ in Theorem 1.1 is also a positive solution to (1.2). But there may be other solutions. 
(2) If $2 q=p+1$ and there is a $m \in \mathbb{N}$ satisfies $3 \leq m<n, p=\frac{m+2}{m-2}$, and $q=\frac{m}{m-2}$, then by Theorem 1.2, we know that there is a solution $v=v\left(x_{1}, \cdots, x_{m-1}, t\right)$ solves

$$
\begin{cases}\Delta v=m(m-2) v^{p}, v>0 & \text { in } H, \\ \frac{\partial v}{\partial t}=-c v^{q} & \text { on } \partial H .\end{cases}
$$

Then $u\left(x_{1}, \cdots, x_{m-1}, \cdots, x_{n-1}, t\right)=v\left(x_{1}, \cdots, x_{m-1}, t\right)$ satisfies equation (1.2) after suitable scaling. But there may be other solutions.

The solution set is more complicated in the case $1 \leq p=q<\frac{n}{n-2}$.

Theorem 1.1 will be proved by applying the standard moving plane method for the equation after Kelvin transformation. The noncritical power yields extra weight functions in the equation and/or on the boundary condition. In return, we can prove that all positive solutions only depend on the variable $t$.

However, the method of moving planes is not suitable for the critical case, basically due to the fact that the reflection plane may stop at any position. Instead, we will use the method of moving spheres, introduced by $\mathrm{Li}$ and $\mathrm{Zhu}$ in [7]. Here, the major difference between our current work and other previous papers using the method of moving spheres is that both scenarios can happen: (1) all reflective spheres never stop, this leads to the result that the solution only depends on $t ;(2)$ all reflective spheres stop at a critical position, this leads to the conclusion that the solution is radially symmetric with respect to a point.

Our paper is organized as follows: We will prove Theorem 1.1 by using the method of moving planes in Section 2. The proof of Theorem 1.2 will be presented in Section 3. In this section, we first consider the case that all reflective spheres do not stop (Proposition 3.3 below), which leads to the solutions that only depend on the last variable; then we consider the case that all reflective spheres do stop at certain points, which leads to the radially symmetric solutions.

\section{NonCRITICAL RESULTS}

In this section, we shall prove Theorem 1.1. For the sake of convenience, after rescaling we can consider the following problem:

$$
\left\{\begin{array}{l}
\Delta u=u^{p}, \quad u>0 \text { in } H, \\
\frac{\partial u}{\partial t}=-u^{q} \text { on } \partial H .
\end{array}\right.
$$

And throughout this section, we always assume that $n \geq 3, p \geq \frac{n+2}{n-2}$ and $1 \leq q<$ $\frac{n}{n-2}$.

Since there is no assumption on the decay rate of $u(x)$ at infinity, as usual we perform the Kelvin transformation on $u$, that is, set

$$
v(x)=\frac{1}{|x|^{n-2}} u\left(\frac{x}{|x|^{2}}\right) .
$$

Then $v(x)$ satisfies

$$
\left\{\begin{array}{l}
\Delta v=|x|^{\tau} v^{p}, \quad v(x)>0 \quad \text { in } H, \\
\frac{\partial v}{\partial t}=-\frac{1}{|x|^{\alpha}} v^{q} \quad \text { on } \partial H \backslash\{0\},
\end{array}\right.
$$


where $\tau=p(n-2)-(n+2)>0$ and $\alpha=n-q(n-2)>0$. For $R>0$, denote

$$
\begin{aligned}
& B_{R}\left(x_{0}\right)=\left\{x \in \mathbb{R}^{n}|| x-x_{0} \mid<R, x_{0} \in \mathbb{R}^{n}\right\}, \\
& B_{R}^{+}\left(x_{0}\right)=\left\{x|| x-x_{0} \mid<R, x \in H, x_{0} \in H\right\} .
\end{aligned}
$$

When $x_{0}=0$, we simply denote $B_{R}=B_{R}(0), B_{R}^{+}=B_{R}^{+}(0)$.

Our goal is to obtain some symmetric properties for $v(x)$. We shall achieve this goal by using the method of moving planes.

Our first lemma, which is a modification of Lemma 2.1 in [7] and Lemma $2.2 \mathrm{in}$ [8, will be used to handle the possible singular point.

Lemma 2.1. Let $v \in C^{2}(H) \cap C^{1}(\bar{H}) \backslash\{0\}$ satisfy (2.2). Then for any $0<\epsilon<$ $\min \left\{1, \min _{\partial B_{1}^{+} \cap \partial B_{1}} v\right\}$, we have $v(x) \geq \frac{\epsilon}{2}$ for all $x \in \overline{B_{1}^{+}} \backslash\{0\}$.

Proof. For $0<r<1$, we introduce an auxiliary function

$$
\varphi(x)=\frac{\epsilon}{2}-\frac{r^{n-2} \epsilon}{|x|^{n-2}}+\frac{\epsilon t^{2}}{2}, \quad x \in B_{1}^{+} \backslash B_{r}^{+},
$$

and let $F_{1}(x)=v(x)-\varphi(x)$. Then we have

$$
\left\{\begin{array}{l}
\Delta F_{1}=|x|^{\tau} v^{p}-\epsilon \quad \text { in } B_{1}^{+} \backslash B_{r}^{+}, \\
\frac{\partial F_{1}}{\partial t}=-\frac{1}{|x|^{\alpha}} v^{q} \quad \text { on } \partial\left(\overline{B_{1}^{+}} \backslash \overline{B_{r}^{+}}\right) \cap \partial \mathbb{R}_{+}^{n} .
\end{array}\right.
$$

We want to show that

$$
F_{1} \geq 0 \quad \text { in } \overline{B_{1}^{+}} \backslash \overline{B_{r}^{+}} .
$$

Let

$$
\begin{aligned}
& S=\left\{x:|x|^{\tau} v^{p}-\epsilon>0\right\} \cap\left(B_{1} \backslash B_{r}\right), \\
& S^{c}=\left\{x:|x|^{\tau} v^{p}-\epsilon \leq 0\right\} \cap\left(B_{1} \backslash B_{r}\right) .
\end{aligned}
$$

In $S$, since $1 \geq|x|>0$, we have $v^{p}(x)>\epsilon /|x|^{\tau} \geq \epsilon$. It follows that $v(x)>$ $\epsilon \geq \varphi_{1}(x)$ for $x \in S$, that is, $F_{1}>0$ in $S$; in $S^{c}, \Delta F_{1} \leq 0$. On $\partial B_{r}^{+} \cap \partial B_{r}$, $F_{1}=v-\left(\frac{\epsilon}{2}-\epsilon+\frac{\epsilon}{2} t^{2}\right) \geq v>0$; on $\partial B_{1}^{+} \cap \partial B_{1}, F_{1}=v-\left(\frac{\epsilon}{2}-r^{n-2} \epsilon+\frac{\epsilon}{2} t^{2}\right)>v-\epsilon \geq 0$. Suppose that (2.5) fails, it follows from the Maximum Principle that there exists some $x_{0}=\left(x_{0}^{\prime}, 0\right)$ with $r<\left|x_{0}^{\prime}\right|<1$ such that $F_{1}\left(x_{0}\right)=\min \overline{B_{1}^{+} \backslash} \overline{B_{r}^{+}} F_{1}<0$. Therefore $\frac{\partial F_{1}}{\partial t}\left(x_{0}\right) \geq 0$, which contradicts to the boundary condition in (2.4). We thus obtain (2.5). Sending $r \rightarrow 0$, we complete the proof of Lemma 2.1

Corollary 2.2. (scaled version). Let $v \in C^{2}(H) \cap C^{1}(\bar{H}) \backslash\{0\}$ solve (2.2). Then for all $0<\epsilon<\min \left\{r_{0}{ }^{(\tau+2) /(1-p)}, \min _{\partial B_{r_{0}}^{+} \cap \partial B_{r_{0}}} v\right\}$, we have $v(x) \geq \frac{\epsilon}{2}$ for all $x \in \overline{B_{r_{0}}^{+}} \backslash\{0\}$.

Proof. For $r_{0}>0$, we obtain the result by applying Lemma 2.1 to $\bar{v}(x)=$ $r_{0}{ }^{(\tau+2) /(p-1)} v\left(r_{0} x\right)$ in $\overline{B_{1}^{+}} \backslash\{0\}$.

For $\lambda<0$, we define

$$
\begin{aligned}
& \Sigma_{\lambda}=\left\{x \mid t>0, x_{1}>\lambda\right\}, T_{\lambda}=\left\{x \mid t \geq 0, x_{1}=\lambda\right\}, \\
& \tilde{\Sigma}_{\lambda}=\bar{\Sigma}_{\lambda} \backslash\{0\}, x^{\lambda} \text { as the reflection point of } x \text { about } T_{\lambda}, \\
& v_{\lambda}(x)=v\left(x^{\lambda}\right), w_{\lambda}=v(x)-v_{\lambda}(x) .
\end{aligned}
$$


Then $w_{\lambda}(x)$ satisfies

$$
\left\{\begin{array}{l}
\Delta w_{\lambda}=|x|^{\tau} v^{p}-\left|x^{\lambda}\right|^{\tau} v_{\lambda}^{p} \leq c_{1}(x) w_{\lambda} \quad \text { in } \Sigma_{\lambda}, \\
\frac{\partial w_{\lambda}}{\partial t}=-\frac{1}{|x|^{\alpha}} v^{q}+\frac{1}{\left|x^{\lambda}\right|^{\alpha}} v_{\lambda}^{q} \leq-c_{2}(x) w_{\lambda} \text { on } \partial H \cap \tilde{\Sigma}_{\lambda},
\end{array}\right.
$$

where $c_{1}(x)=p|x|^{\tau} \cdot \xi_{1}^{p-1}(x)>0, c_{2}(x)=\frac{q}{\left|x^{\lambda}\right|^{\alpha}} \xi_{2}^{q-1}(x)>0, \xi_{1}$ and $\xi_{2}$ are two functions between $v_{\lambda}$ and $v$. Now we are ready to use the moving plane method.

Proposition 2.3. There is a $N>0$, such that, for $\lambda<-N, w_{\lambda}(x) \geq 0$ for all $x \in \tilde{\Sigma}_{\lambda}$.

Proof. Write $z=x+(0, \ldots, 0,1)$. For a fixed positive parameter $\beta \in(0, n-2)$, we define

$$
\varphi_{\lambda}(x)=\left|\left(x_{1}, \ldots, x_{n-1}, t+1\right)\right|^{\beta} w_{\lambda}(x)=|z|^{\beta} w_{\lambda}(x) .
$$

It is sufficient to prove the proposition for $\varphi_{\lambda}(x)$.

A direct calculation shows that

$$
-\triangle \varphi_{\lambda}+\frac{2 \beta}{|z|^{2}} z \cdot \nabla \varphi_{\lambda}+c_{3}(x) \varphi_{\lambda} \geq 0, \quad \text { in } \Sigma_{\lambda},
$$

where $c_{3}(x)=\frac{\beta(n-2-\beta)}{|z|^{2}}+c_{1}(x)>0 ;$ And

$$
\frac{\partial \varphi_{\lambda}}{\partial t}=\frac{\beta}{|z|^{2}} \varphi_{\lambda}+|z|^{\beta} \frac{\partial w_{\lambda}}{\partial t} \leq c_{4}(x) \varphi_{\lambda}, \quad \text { on } \partial H \cap \tilde{\Sigma}_{\lambda}
$$

where

$$
c_{4}(x)=\frac{\beta}{|z|^{2}}-c_{2}(x)=\frac{\beta}{|z|^{2}}-\frac{q}{\left|x^{\lambda}\right|^{\alpha}} \xi_{2}^{q-1}(x) .
$$

Suppose that $\inf _{\Sigma_{\lambda}} \varphi_{\lambda}(x)<0$ for $\lambda$ sufficiently negative. First we observe that

$$
\varphi_{\lambda}(x) \rightarrow 0 \text { as }|x| \rightarrow \infty .
$$

And by Lemma 2.1. we know for some $|\lambda|$ large enough, $w_{\lambda}(x) \geq \frac{\epsilon}{2}>0$ as $x \epsilon$ $\overline{B_{1}^{+}(0)} \backslash\{0\}$. Hence, there exists $\bar{x}$ such that $\varphi_{\lambda}(\bar{x})=\min _{x \in \tilde{\Sigma}_{\lambda}} \varphi_{\lambda}(x)<0$. We know from the Maximum Principle that $\bar{x} \in \partial H \cap \tilde{\Sigma}_{\lambda}$.

Since $\varphi_{\lambda}(\bar{x})<0$, we have

$$
0<v(\bar{x})<\xi_{2}(\bar{x})<v_{\lambda}(\bar{x})=\frac{1}{\left|\tilde{x}^{\lambda}\right|^{n-2}} u\left(\frac{\bar{x}^{\lambda}}{\left|\bar{x}^{\lambda}\right|^{2}}\right)<\frac{A+\circ(1)}{|\lambda|^{n-2}},
$$

where $A=\lim _{|x| \rightarrow 0} u(x)=u(0)$. It follows that

$$
0<c_{2}(\bar{x})<\frac{q(A+\circ(1))^{q-1}}{|\lambda|^{(n-2)(q-1)+\alpha}} \rightarrow 0 \text { as } \lambda \rightarrow-\infty .
$$

Thus $c_{4}(\bar{x})>0$. But this leads to a contradiction to the boundary condition in (2.8).

We then can define

$$
\lambda_{0}=\sup \left\{\lambda<0 \mid w_{\mu}(x) \geq 0 \text { in } \tilde{\Sigma}_{\mu} \text { for all }-\infty<\mu<\lambda\right\} .
$$

Proposition 2.4. $\lambda_{0}=0$ 
Proof. Suppose for the contrary that $\lambda_{0}<0$, then we claim that

$$
w_{\lambda_{0}} \equiv 0 \text {. }
$$

This is a contradiction to the boundary condition in (2.6) since $\alpha>0$. Therefore it suffices to prove (2.11) under the assumption $\lambda_{0}<0$.

Suppose that (2.11) is false, then $w_{\lambda_{0}} \not \equiv 0$ satisfies

$$
\begin{cases}-\Delta w_{\lambda_{0}}+c_{1}(x) w_{\lambda_{0}} \geq 0 & \text { in } \Sigma_{\lambda_{0}}, \\ \frac{\partial w_{\lambda_{0}}}{\partial t} \leq-c_{2}(x) w_{\lambda_{0}} & \text { on } \partial H \cap \tilde{\Sigma}_{\lambda_{0}}, \\ w_{\lambda_{0}} \geq 0 & \text { in } \tilde{\Sigma}_{\lambda_{0}} .\end{cases}
$$

where $c_{1}(x), c_{2}(x)>0$ are the same as that in (2.6). It follows from the Strong Maximum Principle and Hopf lemma that

$$
\begin{cases}w_{\lambda_{0}}(x)>0, & x \in \tilde{\Sigma}_{\lambda_{0}} \backslash T_{\lambda_{0}}, \\ \frac{\partial w_{\lambda_{0}}}{\partial x_{1}}(x)>0, & x \in T_{\lambda_{0}} \cap H .\end{cases}
$$

The following lemma is needed to deal with the possible singular point.

Lemma 2.5. For $r_{0} \leq \min \left\{\frac{1}{2}\left|\lambda_{0}\right|, 1\right\}$, there exists some positive constant $\gamma$ depending only on $\lambda_{0}$ and $r_{0}$ such that $w_{\lambda_{0}}(x)>\gamma$ in $B_{r_{0}}^{+} \backslash\{0\}$.

We also need next lemma to show that negative minimum point of $w_{\lambda}$ for $\lambda<0$ will stay uniformly bounded. We postpone the proofs of both lemmas till the end of the proof of this proposition.

Lemma 2.6. For any fixed $0<\delta<-\lambda_{0}$, there exists an $R_{0}>1$ depending only on $n, q, v$, such that if for any $\lambda \in\left(\lambda_{0}, \lambda_{0}+\delta\right), w_{\lambda}\left(x^{0}\right)=\min _{\tilde{\Sigma}_{\lambda}} w_{\lambda}<0$, then $\left|x^{0}\right| \leq R_{0}$.

We now continue the proof of Proposition 2.4 By the definition of $\lambda_{0}$, we know that there exists a sequence $\lambda_{k} \rightarrow \lambda_{0}$ with $\lambda_{k}>\lambda_{0}$ such that

$$
\inf _{\tilde{\Sigma}_{\lambda_{k}}} w_{\lambda_{k}}<0 \text {. }
$$

Clearly, $\lim _{|x| \rightarrow+\infty} w_{\lambda_{k}}(x)=0$. From Lemma 2.5 and the continuity of $v(x)$ away from the origin, we know that for $k$ large enough, there exists $\gamma>0$ such that

$$
w_{\lambda_{k}}(x)>\frac{\gamma}{2} \text { in } B_{r_{0}}^{+} \backslash\{0\} .
$$

It follows that there exists a point $x^{k}=\left(\left(x^{k}\right)^{\prime}, t^{k}\right) \in \tilde{\Sigma}_{\lambda_{k}} \backslash B_{r_{0}}^{+}$, such that

$$
w_{\lambda_{k}}\left(x^{k}\right)=\min _{\tilde{\Sigma}_{\lambda_{k}}} w_{\lambda_{k}}<0 .
$$

Moreover, Lemma 2.6 implies $\left|x^{k}\right| \leq R_{0}$. From equation (2.6) and the Maximum Principle, we know that $x^{k}$ cannot be a interior point. Thus $x^{k}$ must be on the lateral boundary:

$$
\left\{\left(x_{1}, \ldots, x_{n-1}, t\right): t=0, x_{1}>\lambda_{k}, r_{0} \leq|x| \leq R_{0}\right\} .
$$

Hence,

$$
\frac{\partial w_{\lambda_{k}}}{\partial x_{1}}\left(x^{k}\right)=0, \quad \text { (since along } x_{1} \text { direction is the tangential direction). }
$$


Therefore, there is a sequence of $x^{k}$, still denoted by $x^{k}$, such that $\lim _{k \rightarrow \infty} x^{k}=x^{0}$. By the continuity of $w_{\lambda}$, it holds that $0 \leq w_{\lambda_{0}}\left(x^{0}\right)=\lim _{k \rightarrow \infty} w_{\lambda_{k}}\left(x^{k}\right) \leq 0$, that is, $w_{\lambda_{0}}\left(x^{0}\right)=0$, thus $x^{0}=\left(x_{1}^{0}, \ldots, x_{n-1}^{0}, t^{0}\right) \in T_{\lambda_{0}} \cap\{t=0\}$. From (2.15), we have

$$
w_{\lambda_{0}}\left(x^{0}\right)=0, \quad \frac{\partial w_{\lambda_{0}}}{\partial x_{1}}\left(x^{0}\right)=0
$$

It follows that from (2.13) and (2.16) that $\left|x_{1}^{0}\right|=\lambda_{0}$ and $t^{0}=0$.

We need another lemma to handle the partial derivative about the first variable $x_{1}$ at the corner point.

Lemma 2.7. Suppose (2.12) and (2.13) hold, then $\frac{\partial w_{\lambda_{0}}}{\partial x_{1}}\left(x^{0}\right)>0$ for all $x^{0}=$ $\left(x_{1}^{0}, \ldots, x_{n-1}^{0}, 0\right)$ with $x_{1}^{0}=\lambda_{0}$.

Using Lemma 2.7, we reach a contradiction due to (2.16).

We are left to prove above three lemmas.

Proof of Lemma 2.5. For $0<r<r_{0}, x \in B_{r_{0}}^{+} \backslash B_{r}^{+}$and $\varepsilon>0$ being a positive constant satisfying $\min _{\partial B_{r_{0}}^{+}} w_{\lambda_{0}} \geq \varepsilon$, let $\varphi(x)$ be defined in (2.3), and $F_{2}(x)=$ $w_{\lambda_{0}}(x)-\varphi(x)$. Then $F_{2}(x)$ satisfies

$$
\begin{cases}\Delta F_{2}=|x|^{\tau} v^{p}-\left|x^{\lambda_{0}}\right|^{\tau} v_{\lambda_{0}}^{p}-\epsilon, & x \in B_{r_{0}}^{+} \backslash B_{r}^{+} \\ \frac{\partial F_{2}}{\partial t}=-\frac{1}{|x|^{\alpha}} v^{q}(x)+\frac{1}{\left|x^{\lambda_{0}}\right|^{\alpha}} v_{\lambda_{0}}^{q}(x), & x \in \partial\left(\overline{B_{r_{0}}^{+}} \backslash \overline{B_{r}^{+}}\right) \cap \partial H .\end{cases}
$$

Let

$$
\begin{aligned}
& S=\left\{x:|x|^{\tau} v^{p}-\left|x^{\lambda_{0}}\right|^{\tau} v_{\lambda_{0}}^{p}-\epsilon>0\right\} \cap\left(B_{r_{0}}^{+} \backslash B_{r}^{+}\right), \\
& S^{c}=\left\{x:|x|^{\tau} v^{p}-\left|x^{\lambda_{0}}\right|^{\tau} v_{\lambda_{0}}^{p}-\epsilon \leq 0\right\} \cap\left(B_{r_{0}}^{+} \backslash B_{r}^{+}\right) .
\end{aligned}
$$

In $S,|x|^{\tau}\left(v^{p}-v_{\lambda_{0}}^{p}\right) \geq|x|^{\tau} v^{p}-\left|x^{\lambda_{0}}\right|^{\tau} v_{\lambda_{0}}^{p}>\varepsilon$. It follows from $|x|^{\tau}\left(v^{p}-v_{\lambda_{0}}^{p}\right)=$ $p|x|^{\tau} \cdot \xi_{1}^{p-1}(x) w_{\lambda_{0}}$ that $w_{\lambda_{0}}>\gamma$ for some $\gamma>0$ depending on $r_{0}$ and $\lambda_{0}$.

On the other hand, in $S^{c}, \Delta F_{2} \leq 0$. On $\partial B_{r_{0}}^{+} \cap \partial B_{r_{0}}, F_{2}(x) \geq \varepsilon-\left(\frac{\varepsilon}{2}-\frac{r^{n-2} \varepsilon}{|x|^{n-2}}+\right.$ $\left.\frac{\varepsilon t^{2}}{2}\right)>0$; on $\partial B_{r}^{+} \cap \partial B_{r}, F_{2}(x) \geq \varepsilon-\left(\frac{\varepsilon}{2}-\varepsilon+\frac{\varepsilon t^{2}}{2}\right)>0$. Suppose there exists $x_{0} \in S^{c}$, such that $F_{2}\left(x_{0}\right)=\min F_{2}(x)<0$, then from the Maximum Principle we know that $x_{0} \in \partial\left(B_{r_{0}}^{+} \backslash B_{r}^{+}\right) \cap \partial H$. Then $\frac{\partial F_{2}}{\partial t}\left(x_{0}\right) \geq 0$, which contradicts to the boundary condition of (2.17). Thus $F_{2} \geq 0$ in $S^{c}$ also.

Sending $r \rightarrow 0$, we obtain Lemma 2.5 .

Proof of Lemma 2.6. Choose a test function

$$
\phi(x)=(n-2)^{(n-2) / 2}\left(\frac{\sigma}{\left|x^{\prime}\right|^{2}+(t+\sigma)^{2}}\right)^{(n-2) / 2},
$$

where $\sigma$ satisfies $(n-2) \sigma>q \gamma_{0}^{q-1}$, and $\gamma_{0}$ is a positive constant satisfying $1 / \gamma_{0} \leq$ $|x|^{n-2} v(x) \leq \gamma_{0}$ for $|x| \geq 1$. Let $\bar{w}_{\lambda}(x)=\frac{w_{\lambda}(x)}{\phi(x)}$, then it is a straight forward calculation to verify that

$$
-\triangle \bar{w}_{\lambda}-2(\nabla \phi / \phi) \cdot \nabla \bar{w}_{\lambda}+c_{1}(x) \bar{w}_{\lambda} \geq 0, \quad \text { in } \Sigma_{\lambda} \backslash B_{R_{0}}^{+},
$$

where $c_{1}(x)>0$ is the same as that in (2.6). And

$$
\frac{\partial \bar{w}_{\lambda}}{\partial t}=\bar{c}(x) \bar{w}_{\lambda}, \quad \text { on } \quad\left(\tilde{\Sigma}_{\lambda} \backslash B_{R_{0}}^{+}\right) \cap \partial H
$$


where

$$
\bar{c}(x)=g^{n /(n-2)}-\frac{v^{q}(x) /|x|^{\alpha}-v_{\lambda}^{q}(x) /\left|x^{\lambda}\right|^{\alpha}}{w_{\lambda}(x)} \quad \text { on } \partial H \cap\left(\tilde{\Sigma}_{\lambda} \backslash B_{R_{0}}\right) .
$$

One can show that $\bar{c}(x)>0$ on $\partial H \cap\left(\tilde{\Sigma}_{\lambda} \backslash B_{R_{0}}\right)$ when $w_{\lambda}(x)<0$ and $\alpha=$ $n-q(n-2)>0$ (see, for example, Ou 9]). We thus obtain Lemma 2.6 from (2.18) and (2.19) by using the Maximum Principle.

Proof of Lemma 2.7. Without loss of generality, we assume $\lambda_{0}=-1$. Set $\Omega_{1}=\left\{x=\left(x_{1}, \ldots, x_{n-1}, t\right) \mid-1<x_{1}<-1 / 2, x_{2}^{2}+\ldots+x_{n-1}^{2}+t^{2}<a\right\}$ with $0<a \in(0,1)$ to be chosen later. Due to the continuity of $v$ in $H \backslash\{0\}$, there exists a positive constant $C_{1}$ such that

$$
v_{\lambda_{0}}(x)<C_{1}<+\infty \text { for } x \in \bar{\Omega}_{1} .
$$

Let

$$
h_{1}(x)=\varepsilon\left(\frac{1}{x_{1}^{2}}-1\right)\left(\frac{t^{2} \mu}{2}+1-\mu\right), \quad x \in \Omega_{1},
$$

where $0<\varepsilon, \mu<1$ will be chosen later. Let $A_{1}(x)=w_{\lambda_{0}}(x)-h_{1}(x)$. Then $A_{1}(x)$ satisfies the following equation:

$$
\left\{\begin{array}{c}
\Delta A_{1}(x)=|x|^{\tau} v^{p}-\left|x^{\lambda_{0}}\right|^{\tau} v_{\lambda_{0}}^{p}-\Delta h_{1}(x) \quad \text { in } \Omega_{1} \\
\frac{\partial A_{1}}{\partial t}=-\frac{1}{|x|^{\alpha}} v^{q}+\frac{1}{\left|x^{\lambda_{0}}\right|^{\alpha}} v_{\lambda_{0}}^{q} \leq 0 \quad \text { on } \quad \partial \Omega_{1} \cap \partial H,
\end{array}\right.
$$

and

$$
\Delta h_{1}(x)=6 \varepsilon x_{1}^{-4}\left(\frac{t^{2} \mu}{2}+1-\mu\right)+\varepsilon \mu\left(x_{1}^{-2}-1\right) .
$$

For suitably chosen $\varepsilon$ and $\mu$, we want to show

$$
A_{1}(x)=w_{\lambda_{0}}(x)-h_{1}(x) \geq 0, \quad \forall x \in \Omega_{1} .
$$

Using (2.13), we can choose $\varepsilon_{0}>0$ small enough, such that for all $0<\varepsilon<\varepsilon_{0}$, we have $A_{1}(x) \geq 0$ on $\partial \Omega_{1} \cap\left\{\left\{x_{1}=-1 / 2\right\} \cup\left\{x_{2}^{2}+\ldots+x_{n-1}^{2}+t^{2}=a\right\}\right\}$. Also, from the construction of $h_{1}$ we know $A_{1}(x)=0$ on $\partial \Omega_{1} \cap\left\{x_{1}=-1\right\}$. Suppose the contrary to (2.23); there exists some $\bar{x}=\left(\bar{x}_{1}, \ldots, \bar{x}_{n-1}, \bar{t}\right) \in \overline{\Omega_{1}}$ such that

$$
A_{1}(\bar{x})=\min _{\bar{\Omega}_{1}} A_{1}<0 .
$$

From the above and the boundary condition of (2.21), we have $\bar{t}>0, \bar{x}_{2}^{2}+\ldots+$ $\bar{x}_{n-1}^{2}+\bar{t}^{2}<a,-1<\bar{x}_{1}<-1 / 2$. Thus

$$
\Delta A_{1}(\bar{x}) \geq 0 .
$$

From (2.24), we have $v(\bar{x})-v_{\lambda_{0}}(\bar{x})-h_{1}(\bar{x})<0$, and then

$$
v(\bar{x})<C_{2}<+\infty
$$

for some constant $C_{2}$ depending only on $C_{1}$. By (2.20), (2.26) and the Mean Value Theorem we have $|\bar{x}|^{\tau} v^{p}(\bar{x})-\left|\bar{x}^{\lambda_{0}}\right|^{\tau} v_{\lambda_{0}}^{p}(\bar{x}) \leq C_{3} w_{\lambda_{0}}(\bar{x})$ for some positive constant $C_{3}$ depending only on $C_{1}, C_{2}$ and $\lambda_{0}$. Hence, it follows from (2.21), (2.22) and (2.25) that

$$
C_{3} w_{\lambda_{0}}(\bar{x}) \geq \Delta h_{1}(\bar{x})>\varepsilon \mu\left(\frac{1}{x_{1}^{2}}-1\right) .
$$


Again by (2.24),

$$
w_{\lambda_{0}}(\bar{x})<h_{1}(\bar{x})<\varepsilon\left(\frac{1}{x_{1}^{2}}-1\right)\left(\frac{\mu}{2 C_{3}}+1-\mu\right),
$$

where $\bar{t}^{2}<a$, and we have chosen $0<a \leq C_{3}{ }^{-1}$. Therefore, we choose $a \leq$ $\min \left\{C_{3}{ }^{-1}, 1\right\}$ such that both (2.27) and (2.28) hold. Combining (2.27) and (2.28), we have

$$
\varepsilon C_{3}\left(\frac{1}{x_{1}^{2}}-1\right)\left(\frac{\mu}{2 C_{3}}+1-\mu\right)>\varepsilon \mu\left(\frac{1}{x_{1}^{2}}-1\right)
$$

i. e., $\mu<\frac{2 C_{3}}{1+2 C_{3}}$. If $\mu$ is chosen in such a way that $\mu>\frac{2 C_{3}}{1+2 C_{3}}$ from the beginning, we reach a contradiction. Thus (2.23) holds. Since we also know that $A_{1}\left(x^{0}\right)=0$, we have

$$
\frac{\partial A_{1}}{\partial x_{1}}\left(x^{0}\right) \geq 0 .
$$

It follows from a direct computation that

$$
\frac{\partial w_{\lambda_{0}}}{\partial x_{1}}\left(x^{0}\right)=\frac{\partial A_{1}}{\partial x_{1}}\left(x^{0}\right)+\frac{\partial h_{1}}{\partial x_{1}}\left(x^{0}\right) \geq \frac{\partial h_{1}}{\partial x_{1}}\left(x^{0}\right)=2 \varepsilon(1-\mu)>0 .
$$

Hence, Lemma 2.7 is established.

We hereby complete the proof of Proposition 2.4

Proof of Theorem 1.1. From Proposition 2.4 we know that $v\left(-x_{1}, x_{2}, \cdots, t\right) \leq$ $v\left(x_{1}, x_{2}, \cdots, t\right)$ for $x_{1} \geq 0$. Similarly, if we move planes from the positive direction of $x_{1}$, we see that $v\left(-x_{1}, x_{2}, \cdots, t\right) \geq v\left(x_{1}, x_{2}, \cdots, t\right)$ for $x_{1} \geq 0$. Thus, $v\left(x^{\prime}, t\right)$ is symmetric with respect to $x_{1}$. Clearly the above argument can be applied to any direction perpendicular to $t$-axis, therefore we conclude that $v\left(x^{\prime}, t\right)=v\left(\left|x^{\prime}\right|, t\right)$. It follows that $u\left(x^{\prime}, t\right)=u\left(\left|x^{\prime}\right|, t\right)$ due to the inverse Kelvin transformation. Since we can choose the origin arbitrarily on the hyperplane $t=0$, it is easy to see that $u\left(x^{\prime}, t\right)$ is independent of $x^{\prime}$. (1.2) is reduced to the following ordinary differential equation:

$$
\left\{\begin{array}{l}
u^{\prime \prime}(t)=n(n-2) u^{p}(t), \quad u>0, \quad t>0, \\
u^{\prime}(0)=-c_{+} u^{q}(0) .
\end{array}\right.
$$

It is easy to check that (2.29) has the following unique positive solution

$$
u=\left(\frac{p-1}{2} A t+B\right)^{-2 /(p-1)}
$$

where $A=\left(\frac{2 n(n-2)}{p+1}\right)^{1 / 2}$ and $B=\left(c_{+}^{-1} A\right)^{-(p-1) /(2 q-p-1)}$. This completes the proof of Theorem 1.1]

\section{Critical Results}

In this section, we shall deal with the critical case and prove Theorem 1.2.

Assume $u(x) \in C^{2}(H) \cap C^{1}(\bar{H})$ solves (1.2). Throughout this whole section, we assume that $p=\frac{n+2}{n-2}, q=\frac{n}{n-2}($ and $n \geq 3)$.

For any given point $b \in \partial H=\mathbb{R}^{n-1}$, we define the Kelvin transformation of $u$ centered at $b$ by

$$
v_{b}(x)=\frac{1}{|x|^{n-2}} u_{b}\left(\frac{x}{|x|^{2}}\right)
$$


where $u_{b}(x)=u\left(x^{\prime}+b, t\right)$. If $b=0$ is the origin, for simplicity, we denote $v(x)=$ $v_{0}(x)$. Then $v(x)$ satisfies

$$
\left\{\begin{array}{l}
\Delta v=n(n-2) v^{p}, \quad v(x)>0 \quad \text { in } H \\
\frac{\partial v}{\partial t}=-c_{+} v^{q} \quad \text { on } \quad \partial H \backslash\{0\} .
\end{array}\right.
$$

For $\lambda>0$ and $b \in \partial H$, define

$$
w_{\lambda, b}(x)=v_{b}(x)-\frac{\lambda^{n-2}}{|x|^{n-2}} v_{b}\left(\frac{\lambda^{2} x}{|x|^{2}}\right) .
$$

In the rest of this section, we always write $v_{\lambda}(x)=\left(\lambda^{n-2} /|x|^{n-2}\right) v\left(\lambda^{2} x /|x|^{2}\right)$, $w_{\lambda}(x)=v(x)-v_{\lambda}(x)$. Clearly, $w_{\lambda}$ satisfies

$$
\left\{\begin{array}{l}
\Delta w_{\lambda}=n(n-2)\left(v^{p}-v_{\lambda}^{p}\right)=c_{5}(x) w_{\lambda} \quad \text { in } B_{\lambda}^{+}, \\
\frac{\partial w_{\lambda}}{\partial t}=-c_{+}\left(v^{q}-v_{\lambda}^{q}\right)=-c_{6}(x) w_{\lambda} \quad \text { on } \quad \partial B_{\lambda}^{+} \cap \partial H \backslash\{0\},
\end{array}\right.
$$

where $c_{5}(x)=n(n+2) \xi_{3}(x)^{4 /(n-2)}, c_{6}(x)=c_{+}(n-2)^{-1} n \xi_{4}(x)^{2 /(n-2)}, \xi_{3}$ and $\xi_{4}$ are two functions between $v_{\lambda}$ and $v$.

We need to establish a lemma which will be used to handle the possible singular point.

Lemma 3.1. Let $v \in C^{2}(H) \cap C^{1}(\bar{H}) \backslash\{0\}$ solve (3.1). Then for any $0<\epsilon<$ $\min \left\{R^{(2-n) / 2}, \min _{\partial B_{R}^{+} \cap \partial B_{R}} v\right\}$, we have $v(x) \geq \frac{\epsilon}{2}$ for all $x \in \overline{B_{R}^{+}} \backslash\{0\}$.

Proof. When $R=1$, this lemma can be proved as that of Lemma 2.1 with $\tau=\alpha=0$. For more general $R$, it follows easily from applying the result for $R=1$ to $\bar{v}(x)=R^{(n-2) / 2} v(R x)$.

Next we will prove Theorem 1.2 by the moving sphere method.

First we establish a proposition which makes it possible to start moving the spheres.

Proposition 3.2. For $\lambda$ large enough, $w_{\lambda}(x) \geq 0$ for all $x \in B_{\lambda}^{+} \backslash\{0\}$.

Proof. We prove this proposition by three steps as that in 7 .

Step 1. Similar to the proof of Proposition 2.1 in [7, we have: there exists $R_{0}>0$ large enough, such that for all $R_{0} \leq|x| \leq \lambda / 2, w_{\lambda}(x) \geq 0$.

Step 2. Let $R_{1} \geq R_{0}$ and $R_{1} \leq \lambda / 2 \leq|x| \leq \lambda$. We claim that $w_{\lambda}(x) \geq 0$. To see this, we define

$$
\varphi_{\lambda}(x)=|y|^{\beta} w_{\lambda}(x)
$$

for $\beta \in(0, n-2), y=x+(0, \ldots, 0, \lambda / 4)$. Easy to check that $\varphi_{\lambda}$ satisfies

$$
\left\{\begin{array}{l}
-\Delta \varphi_{\lambda}+\frac{2 \beta}{|y|^{2}} y \cdot \nabla \varphi_{\lambda}+\left(c_{5}(x)+\frac{\beta(n-2-\beta)}{|y|^{2}}\right) \varphi_{\lambda}=0, \quad \text { in } B_{\lambda}^{+}, \\
\frac{\partial \varphi_{\lambda}}{\partial t}=\left(\frac{\beta \lambda}{4|y|^{2}}-c_{6}(x)\right) \varphi_{\lambda} \quad \text { on } \partial B_{\lambda}^{+} \cap \partial H \backslash\{0\} .
\end{array}\right.
$$

If there exists $x_{0}$ with $\lambda / 2 \leq\left|x_{0}\right| \leq \lambda$, such that $\varphi_{\lambda}\left(x_{0}\right)=\min _{\lambda / 2 \leq|x| \leq \lambda} \varphi_{\lambda}(x)<0$, then from step 1 and the definition of $w_{\lambda}$, we know $x_{0} \notin\{|x|=\lambda / 2\} \cup\{|x|=\lambda\}$. The Maximum Principle yields that $x_{0} \in \partial H$. As in the proof of Proposition 2.1 in 
[7, we can show that $c_{6}\left(x_{0}\right) \leq C /\left|x_{0}\right|^{2}$ with $C>0$. Moreover, for $\lambda$ large enough and $y_{0}=x_{0}+(0, \ldots, 0, \lambda / 4)$, we have

$$
\frac{\beta \lambda}{4\left|y_{0}\right|^{2}}-c_{6}\left(x_{0}\right)>0, \quad x_{0} \in \partial H .
$$

This contradicts to the boundary condition in (3.3).

Step 3. We claim: there exists $R_{2} \geq R_{1}$, such that for $\lambda \geq R_{2}, w_{\lambda}(x) \geq 0$ for $x \in B_{R_{0}}^{+} \backslash\{0\}$.

From the fact that $\lim _{|y| \rightarrow+\infty}|y|^{n-2} v(y)=u(0),\left|\frac{\lambda^{2} x}{|x|^{2}}\right| \geq \frac{\lambda^{2}}{R_{0}} \geq \frac{R_{2}^{2}}{R_{0}}$ and Lemma 3.1 we have

$$
w_{\lambda}(x)=v(x)-\frac{1}{\lambda^{n-2}}\left(\left|\frac{\lambda^{2} x}{|x|^{2}}\right|^{n-2} v\left(\frac{\lambda^{2} x}{|x|^{2}}\right)\right)>\frac{\varepsilon}{2}-\frac{u(0)+\circ(1)}{\lambda^{2}} .
$$

So Step 3 follows easily as $\lambda$ becomes large. Proposition 3.2 is proved.

Now for any $b \in \partial H=\mathbb{R}^{n-1}$, we define

$$
\lambda_{b}=\inf \left\{\lambda>0 \mid w_{\mu, b}(x) \geq 0 \text { in } \overline{B_{\mu}^{+}} \backslash\{0\} \text { for all } \lambda<\mu<\infty\right\} .
$$

Proposition 3.3. Assume $u(x) \in C^{2}(H) \cap C^{1}(\bar{H})$ solves (1.2). If $\lambda_{b}=0$ for all $b \in \partial H=\mathbb{R}^{n-1}$, then $c_{+}=n-2$ and $u(x)=u\left(x^{\prime}, t\right)=u(0, t)=u(t)=$ $\left(2 t+u(0)^{-2 /(n-2)}\right)^{-(n-2) / 2}$, for all $x=\left(x^{\prime}, t\right) \in H$.

In order to prove Proposition 3.3, we need the following technical Li-Zhu lemma which appeared first in [7, Lemma 2.2].

Lemma 3.4. (Lemma 2.2 in [7]) Suppose $f \in C^{1}(H)$ satisfies: for any $b \in \partial H, \lambda>$ 0

$$
f_{b}(x)-\frac{\lambda^{n-2}}{|x|^{n-2}} f_{b}\left(\frac{\lambda^{2} x}{|x|^{2}}\right) \geq 0, \quad \forall x \in B_{\lambda}^{+},
$$

where $f_{b}(x)=f\left(x^{\prime}+b, t\right), \forall x=\left(x^{\prime}, t\right) \in H$. Then $f(x)=f\left(x^{\prime}, t\right)=f(0, t)$ for all $x \in H$.

Proof of Proposition 3.3. If $\lambda_{b}=0$ for all $b \in \partial H=\mathbb{R}^{n-1}$, then $u\left(x^{\prime}, t\right) \in$ $C^{1}(\bar{H})$ satisfies (3.4). We know from Lemma 3.4 that $u(x)=u\left(x^{\prime}, t\right)=u(0, t)=$ $u(t)$, thus

It is easy to see that

$$
\left\{\begin{array}{l}
u^{\prime \prime}(t)=n(n-2) u^{p}(t), \quad u>0, \quad t>0, \\
u^{\prime}(0)=-c_{+} u^{q}(0) .
\end{array}\right.
$$

$$
\left(u^{\prime}(t)\right)^{2}=(n-2)^{2} u^{2 n /(n-2)}(t)+\left(c_{+}^{2}-(n-2)^{2}\right) u^{2 n /(n-2)}(0) .
$$

When $c_{+}=n-2, u(t)$ satisfies

$$
\left\{\begin{array}{l}
\left(u^{\prime}(t)\right)^{2}=(n-2)^{2} u^{2 n /(n-2)}(t), \quad u>0, \quad t>0, \\
u^{\prime}(0)=-(n-2) u^{2 n /(n-2)}(0),
\end{array}\right.
$$

We then obtain $u(t)=\left(2 t+u(0)^{-2 /(n-2)}\right)^{-(n-2) / 2}$.

When $c_{+}>n-2$, it holds

$$
u^{\prime}(t)<-\sqrt{\left(c_{+}^{2}-(n-2)^{2}\right) u^{2 n /(n-2)}(0)},
$$

then there is no global positive solution. 
When $0<c_{+}<n-2$, since $u^{\prime \prime}(t)>0$, we know that $u(t)$ satisfies

$$
u^{\prime}(t)=\left\{\begin{array}{lc}
-\sqrt{(n-2)^{2} u^{\frac{2 n}{n-2}}(t)+\left(c_{+}^{2}-(n-2)^{2}\right) u^{\frac{2 n}{n-2}}(0)}, & 0 \leq t<t_{0}, \\
0, & t=t_{0}, \\
\sqrt{(n-2)^{2} u^{\frac{2 n}{n-2}}(t)+\left(c_{+}^{2}-(n-2)^{2}\right) u^{\frac{2 n}{n-2}}(0),} & t>t_{0},
\end{array}\right.
$$

for some positive $t_{0}$. This means $u(t) \rightarrow+\infty$ as $t \rightarrow+\infty$. Thus there exists $t_{1}>t_{0}$, such that for $t>t_{1},(n-2)^{2} u^{\frac{2 n}{n-2}}(t)-\left((n-2)^{2}-c_{+}^{2}\right) u^{\frac{2 n}{n-2}}(0)>\frac{1}{2}(n-2)^{2} u^{\frac{2 n}{n-2}}(t)$. So, we have that for $t>t_{1}$,

$$
\begin{aligned}
t-t_{1} & =\int_{t_{1}}^{t} \frac{u^{\prime}(s) d s}{\sqrt{(n-2)^{2} u^{\frac{2 n}{n-2}}(s)+\left(c_{+}^{2}-(n-2)^{2}\right) u^{\frac{2 n}{n-2}}(0)}} \\
& =\int_{u\left(t_{1}\right)}^{u(t)} \frac{d u}{\sqrt{(n-2)^{2} u^{\frac{2 n}{n-2}}+\left(c_{+}^{2}-(n-2)^{2}\right) u^{\frac{2 n}{n-2}}(0)}} \\
& \leq \int_{u\left(t_{1}\right)}^{u(t)} \frac{\sqrt{2} d u}{(n-2) u^{\frac{n}{n-2}}} \\
& =\frac{\sqrt{2}}{2} u^{-2 /(n-2)}\left(t_{1}\right)-\frac{\sqrt{2}}{2} u^{-2 /(n-2)}(t),
\end{aligned}
$$

which impies

$$
u^{-2 /(n-2)}(t) \leq u^{-2 /(n-2)}\left(t_{1}\right)-\sqrt{2}\left(t-t_{1}\right) .
$$

But as $t \rightarrow+\infty, u^{-2 /(n-2)}\left(t_{1}\right)-\sqrt{2}\left(t-t_{1}\right) \rightarrow-\infty$, contradiction! So there is no positive global solution. We hereby complete the proof of Proposition 3.3 .

Next, we consider the other possibility, that is, $\lambda_{b}>0$ for some $b \in \partial H=\mathbb{R}^{n-1}$.

Proposition 3.5. Assume $u(x) \in C^{2}(H) \cap C^{1}(\bar{H})$ solves (1.2). If $\lambda_{b}>0$ for some $b \in \partial H=\mathbb{R}^{n-1}$, then

$$
w_{\lambda_{b}, b}(x) \equiv 0, \quad \forall x=\left(x^{\prime}, t\right) \in H .
$$

Proof. From the properties of the Kelvin transformation, we only need to prove this proposition for $x \in B_{\lambda_{b}}^{+}(b)$. Without loss of generality, we assume $b=0$. Suppose the contrary to Proposition [3.5, then $w_{\lambda_{0}} \not \equiv 0$ satisfies

$$
\begin{cases}-\Delta w_{\lambda_{0}}+c_{5}(x) w_{\lambda_{0}}=0 & \text { in } B_{\lambda_{0}}^{+} \\ \frac{\partial w_{\lambda_{0}}}{\partial t}=-c_{6}(x) w_{\lambda_{0}} & \text { on } \partial B_{\lambda_{0}}^{+} \cap \partial H \backslash\{0\}, \\ w_{\lambda_{0}} \geq 0 & \text { in } \overline{B_{\lambda_{0}}^{+}} \backslash\{0\} .\end{cases}
$$

where $c_{5}(x), c_{6}(x)>0$ are the same as that in (3.2). It follows from the Strong Maximum Principle and the Hopf lemma that

$$
\begin{cases}w_{\lambda_{0}}(x)>0, & x \in \overline{B_{\lambda_{0}}^{+}}, 0<|x|<\lambda_{0}, \\ \frac{\partial w_{\lambda_{0}}}{\partial \nu}(x)>0, & x \in \partial B_{\lambda_{0}}^{+} \cap H\end{cases}
$$

where $\nu$ denotes the inner normal of the sphere $\partial B_{\lambda_{0}}$. The following lemma is needed to deal with the possible singular point, and we postpone its proof till the end of the proof of the proposition. 
Lemma 3.6. There exists a positive constant $\gamma=\gamma\left(\lambda_{0}\right)>0$ such that $w_{\lambda_{0}}(x)>\gamma$ for $x \in \overline{B_{\lambda_{0} / 2}^{+}} \backslash\{0\}$.

We continue the proof of Proposition 3.5 From the definition of $\lambda_{0}$, we know that there exists a sequence $\lambda_{k} \rightarrow \lambda_{0}$ with $\lambda_{k}<\lambda_{0}$, such that

$$
\underset{B_{\lambda_{k}}^{+} \backslash\{0\}}{\inf _{\lambda_{k}}<0}
$$

From Lemma 3.6 and the continuity of $w_{\lambda}$ away from the origin, it follows that for $k$ large enough, there exists $x^{k}=\left(\left(x^{k}\right)^{\prime}, t^{k}\right) \in \overline{B_{\lambda_{k}}^{+}} \backslash B_{\lambda_{0} / 2}^{+}$such that

$$
w_{\lambda_{k}}\left(x^{k}\right)=\frac{\min }{B_{\lambda_{k}}^{+} \backslash\{0\}} w_{\lambda_{k}}(x)<0 .
$$

It is clear that $\frac{\lambda_{0}}{2}<\left|x^{k}\right|<\lambda_{k}$, And, due to the Strong Maximum Principle, $t^{k}=0$. Hence, $\frac{\partial w_{\lambda_{k}}}{\partial x_{i}}\left(x^{k}\right)=0(i=1, \cdots, n-1)$ and $\frac{\partial w_{\lambda_{k}}}{\partial t}\left(x^{k}\right) \geq 0$. After passing to a subsequence (still denoted as $\left.x^{k}\right), x^{k} \rightarrow x_{0}=\left(x_{0}^{\prime}, 0\right)$. It follows that

$$
w_{\lambda_{0}}\left(x_{0}\right)=0, \quad \frac{\partial w_{\lambda_{0}}}{\partial x_{i}}\left(x_{0}\right)=0,(i=1, \cdots, n-1) .
$$

We know from (3.6) and (3.8) that $\left|x_{0}^{\prime}\right|=\lambda_{0}$.

The following lemma is needed to handle the normal derivative, and we postpone its proof till the end.

Lemma 3.7. Suppose (3.5) and (3.6) hold, then $\frac{\partial w_{\lambda_{0}}}{\partial \nu}\left(x_{0}\right)>0$ for all $x_{0}=\left(x_{0}^{\prime}, 0\right)$ and $\left|x_{0}^{\prime}\right|=\lambda_{0}$.

From Lemma 3.7, we reach a contradiction due to (3.8).

We are left to prove above two lemmas

Proof of Lemma 3.6. Using (3.6), we have $\min _{\partial B_{\lambda_{0} / 2}^{+} \cap \partial B_{\lambda_{0} / 2}} w_{\lambda_{0}} \geq \varepsilon$ for some $0<\varepsilon<1$. Without loss of generality, we assume $\lambda_{0}=2$. For $0<r<1$ and $x \in$ $B_{1}^{+} \backslash B_{r}^{+}$, let $\varphi(x)$ be the same function given in (2.3), and $F_{3}(x)=w_{\lambda_{0}}(x)-\varphi(x)$. Then $F_{3}(x)$ satisfies

$$
\left\{\begin{array}{l}
\Delta F_{3}=n(n-2)\left(v^{p}-v_{\lambda_{0}}^{p}\right)-\epsilon \text { in } B_{1}^{+} \backslash B_{r}^{+}, \\
\frac{\partial F_{3}}{\partial t}=-c_{+}\left(v^{q}-v_{\lambda_{0}}^{q}\right) \quad \text { on } \partial\left(B_{1}^{+} \backslash B_{r}^{+}\right) \cap \partial H .
\end{array}\right.
$$

If $n(n-2)\left(v^{p}-v_{\lambda_{0}}^{p}\right)-\epsilon \geq 0$, we have proved Lemma 3.6 with $\gamma=\frac{\varepsilon}{n(n-2) C}$, $C=\max _{B_{1}^{+} \backslash B_{r}^{+}} v>0$ and $r \rightarrow 0$. Otherwise, it follows from (3.9) that

$$
\left\{\begin{array}{l}
\Delta F_{3}<0 \quad \text { in } B_{1}^{+} \backslash B_{r}^{+} \\
\frac{\partial F_{3}}{\partial t}<0 \quad \text { on } \partial\left(B_{1}^{+} \backslash B_{r}^{+}\right) \cap \partial H
\end{array}\right.
$$

Now, we will show

$$
F_{3} \geq 0 \quad \text { in } \overline{B_{1}^{+}} \backslash B_{r}^{+} \text {. }
$$


On $\partial B_{r}^{+} \cap \partial B_{r}, F_{3}>w_{\lambda_{0}}>0$; on $\partial B_{1}^{+} \cap \partial B_{1}, F_{3} \geq \varepsilon-\left(\frac{\epsilon}{2}-r^{n-2} \epsilon+\frac{\epsilon}{2} t^{2}\right)>0$. Suppose that (3.11) fails, it follows from the Strong Maximum Principle that there exists some $x_{0}=\left(x_{0}^{\prime}, 0\right)$ with $r<\left|x_{0}^{\prime}\right|<1$ such that

$$
F_{3}\left(x_{0}\right)=\min _{\overline{B_{1}^{+} \backslash B_{r}^{+} \cap \partial H}} F_{3}<0,
$$

which contradicts to the boundary condition of (3.10). This establishes (3.11). Sending $r \rightarrow 0$, we obtain Lemma 3.6 .

Proof of Lemma 3.7. Without loss of generality, we assume $\lambda_{0}=1$. Set $\Omega_{2}=$ $\left\{x=\left(x^{\prime}, t\right) \mid x \in B_{1}^{+} \backslash \overline{B_{1 / 2}^{+}}, t<a\right\}$ with $0<a \leq 1 / 2$ to be chosen later. Due to the continuity of $v$ in $H \backslash\{0\}$, we know that: there exists some positive constant $C_{1}$ such that

$$
v_{\lambda_{0}}(x)<C_{1}<+\infty \text { for } x \in \overline{\Omega_{2}} .
$$

Let

$$
h_{2}(x)=\varepsilon\left(\frac{1}{|x|^{n-2}}-1\right)\left(\frac{t^{2} \mu}{2}+\frac{1-\mu}{(2|x|)^{n-2}}+1-\mu\right), \quad x \in \Omega_{2},
$$

where $0<\varepsilon, \mu<1$ will be chosen later. Let $A_{2}(x)=w_{\lambda_{0}}(x)-h_{2}(x)$, then $A_{2}(x)$ satisfies the following equation.

$$
\left\{\begin{aligned}
\Delta A_{2}(x) & =c_{5}(x) w_{\lambda_{0}}(x)-\Delta h_{2}(x) \quad \\
\frac{\partial A_{2}}{\partial t} & =-c_{6}(x) w_{\lambda_{0}}(x) \leq 0 \quad \text { in } \Omega_{2} \\
& \text { on } \quad \partial \Omega_{2} \cap \partial H
\end{aligned}\right.
$$

where $c_{5}(x)$ and $c_{6}(x)$ are given in (3.2), and

$$
\Delta h_{2}(x)=\epsilon\left[\mu\left(\frac{1}{|x|^{n-2}}-1\right)+2(n-2)\left(\frac{(1-\mu)(n-2)}{2^{n-2}|x|^{2 n-2}}-\frac{\mu t^{2}}{|x|^{n}}\right)\right] .
$$

For suitably chosen $\varepsilon$ and $\mu$, we want to show

$$
A_{2}(x)=w_{\lambda_{0}}(x)-h_{2}(x) \geq 0, \quad \forall x \in \Omega_{2} .
$$

Using (3.6), we can choose $\varepsilon_{0}>0$ small enough, such that for all $0<\varepsilon<\varepsilon_{0}$, we have $A_{2}(x) \geq 0$ on $\partial \Omega_{2} \cap\left\{\partial B_{1 / 2} \cup\{t=a\}\right\}$. Also, from the construction of $h_{2}$ we know $A_{2}(x)=0$ on $\partial \Omega_{2} \cap \partial B_{1}$. Suppose the contrary to (3.15), then there exists some $\bar{x}=\left(\bar{x}^{\prime}, \bar{t}\right) \in \bar{\Omega}$ such that

$$
A_{2}(\bar{x})=\min _{\bar{\Omega}_{2}} A_{2}<0 .
$$

From the above and the boundary condition of (3.13), we have $0<\bar{t}<a, 1 / 2<$ $|\bar{x}|<1$. Thus

$$
\Delta A_{2}(\bar{x}) \geq 0 .
$$

From (3.16), we have $v(\bar{x})-v_{\lambda_{0}}(\bar{x})-h_{2}(\bar{x})<0$, and then

$$
v(\bar{x})<C_{2}<+\infty
$$

for some constant $C_{2}$ depending only on $C_{1}$. By (3.12) and (3.18) we have $c_{5}(x)<C_{3}$ for some positive constant $C_{3}$ depending only on $C_{1}, C_{2}$ and $\lambda_{0}$. Hence, it follows from (3.13), (3.14) and (3.17) that

$$
C_{3} w_{\lambda_{0}}(\bar{x}) \geq c_{5}(\bar{x}) w_{\lambda_{0}}(\bar{x})>\varepsilon \mu\left(\frac{1}{|x|^{n-2}}-1\right),
$$


for $\bar{t}<a$ if we choose $0<a \leq\left((1-\mu) \mu^{-1} 2^{2-n}(n-2)\right)^{1 / 2}$ such that $\frac{(1-\mu)(n-2)}{2^{n-2}|x|^{2 n-2}}-$ $\frac{\mu t^{2}}{|x|^{n}}>0$. Again by (3.16),

$$
\begin{aligned}
w_{\lambda_{0}}(\bar{x})<h_{2}(\bar{x}) & <\varepsilon\left(\frac{1}{|x|^{n-2}}-1\right)\left[\frac{\mu \bar{t}^{2}}{2}+2(1-\mu)\right] \\
& <\varepsilon\left(\frac{1}{|x|^{n-2}}-1\right)\left[\frac{\mu}{2 C_{3}}+2(1-\mu)\right],
\end{aligned}
$$

for $\bar{t}<a$ if we choose $0<a \leq C_{3}^{-1 / 2}$. Therefore, if we choose $a \leq \min \{((1-$ н) $\left.\left.\mu^{-1} 2^{2-n}(n-2)\right)^{1 / 2}, C_{3}^{-1 / 2}, 1 / 2\right\}$ such that both (3.19) and (3.20) hold, then

$$
C_{3} \varepsilon\left(\frac{1}{|x|^{n-2}}-1\right)\left[\frac{\mu}{2 C_{3}}+2(1-\mu)\right]>\varepsilon \mu\left(\frac{1}{|x|^{n-2}}-1\right) ;
$$

that is, $\mu<\frac{4 C_{3}}{1+4 C_{3}}$. If $\mu$ is chosen in such a way that $\mu>\frac{4 C_{3}}{1+4 C_{3}}$ from the beginning, we reach a contradiction. Thus (3.15) holds. Since we also know that $A_{2}\left(x_{0}\right)=0$, we have

$$
\frac{\partial A_{2}}{\partial \nu}\left(x_{0}\right) \geq 0
$$

It follows from a direct computation that

$$
\frac{\partial w_{\lambda_{0}}}{\partial \nu}\left(x_{0}\right)=\frac{\partial A_{2}}{\partial \nu}\left(x_{0}\right)+\frac{\partial h_{2}}{\partial \nu}\left(x_{0}\right) \geq \frac{\partial h_{2}}{\partial \nu}\left(x_{0}\right)=\varepsilon(n-2)\left(2^{2-n}+1\right)(1-\mu)>0 .
$$

Hence, Lemma 3.7 is established.

We hereby complete the proof of the proposition.

Proposition 3.8. Suppose $\lambda_{b}>0$ for some $b \in \partial H$, then we have $\lambda_{b}>0$ for all $b \in \partial H$.

Proof. This proposition can be proved in the same way as that for Claim 4 of Proposition 2.1 in [7.

We will also need the second technical Li-Zhu lemma which first appeared in 7. Lemma 2.5]

Lemma 3.9. (Lemma 2.5 in [7]) Suppose $f \in C^{1}(H)(n \geq 3)$ satisfying: for all $b \in \partial H=\mathbb{R}^{n-1} ;$ there exists $\mu_{b} \in \mathbb{R}$ such that

$$
f\left(x^{\prime}+b\right)=\frac{\mu_{b}^{n-2}}{\left|x^{\prime}\right|^{n-2}} f\left(\frac{\mu_{b}^{2} x^{\prime}}{\left|x^{\prime}\right|^{2}}+b\right), \quad \forall x^{\prime} \in \partial H \backslash\{0\} .
$$

Then for some $a \geq 0, d>0, x_{0}^{\prime} \in \partial H$,

$$
f\left(x^{\prime}\right)= \pm\left(\frac{a}{\left|x^{\prime}-x_{0}^{\prime}\right|^{2}+d}\right)^{(n-2) / 2}, \quad \forall x^{\prime} \in \partial H .
$$

It is easy to see from Proposition 3.5. Proposition 3.8 and Lemma 3.9 that

Lemma 3.10. Let $u$ be a positive function satisfying the hypotheses of Theorem 1.2. Suppose $\lambda_{b}>0$ for some $b \in \partial H=\mathbb{R}^{n-1}$, then we have for all $a, d>0, x_{0}^{\prime} \in \partial H$,

$$
u\left(x^{\prime}, 0\right)=\left(\frac{a}{\left|x^{\prime}-x_{0}^{\prime}\right|^{2}+d}\right)^{(n-2) / 2}, \quad \forall x^{\prime} \in \partial H .
$$


Proof of Theorem 1.2. We divide the proof of this theorem into two cases.

Case 1. Suppose $\lambda_{b}=0$ for all $b \in \partial H=\mathbb{R}^{n-1}$, then from Proposition 3.3 we have $u(x)=u\left(x^{\prime}, t\right)=u(0, t)=u(t)$ for $\forall x=\left(x^{\prime}, t\right) \in H$. Moreover, in terms of value range of $c_{+}, u(t)$ has three different expressions as in Proposition 3.3 .

Case 2. Suppose $\lambda_{b}>0$ for some $b \in \partial H=\mathbb{R}^{n-1}$, then from Lemma 3.10 we have for all $a, d>0, x_{0}^{\prime} \in \partial H$,

$$
u\left(x^{\prime}, 0\right)=\left(\frac{a}{\left|x^{\prime}-x_{0}^{\prime}\right|^{2}+d}\right)^{(n-2) / 2}, \quad \forall x^{\prime} \in \partial H .
$$

As in [7, let $x_{0}=\left(x_{0}^{\prime},-\sqrt{d}\right)$, set

$$
\varphi(x)=\frac{1}{\left|x-x_{0}\right|^{n-2}} u\left(\frac{x-x_{0}}{\left|x-x_{0}\right|^{2}}+x_{0}\right),
$$

and $B=\left\{\left(x^{\prime}, t\right) \mid\left[(t+\sqrt{d}) /\left(\left|x^{\prime}-x_{0}^{\prime}\right|^{2}+(t+\sqrt{d})^{2}\right)\right]-\sqrt{d}>0\right\}$. Actually, $B$ is a ball in $\mathbb{R}^{n}$. Without loss of generality, we assume $a=1$ in Lemma 3.9. A direct computation as in [7] shows that $\varphi(x)=1$ on $\partial B$. Moreover, we have

$$
\Delta \varphi(x)=n(n-2) \varphi^{p} \quad \text { in } B .
$$

It is easy to see that the solution to this equation is unique. And it follows from the Strong Maximum Principle that $\varphi<1$ in $B$. Define $\psi(x)=1-\varphi(x)$. Clearly, $\psi$ satisfies

$$
\left\{\begin{aligned}
-\Delta \psi & =n(n-2)(1-\psi)^{p} & & \text { in } B \\
\psi & =0 & & \text { on } \partial B
\end{aligned}\right.
$$

where $0<\psi<1$ in $B$. Applying the result of [3], we know that $\psi$ is radically symmetric about the center of $B$. Hence $\varphi(x)$ must take the form

$$
\varphi(x)=\left(\frac{\varepsilon}{\varepsilon^{2}-\left|x-x_{1}\right|^{2}}\right)^{(n-2) / 2}
$$

for some $\varepsilon>0$ and $x_{1} \in \mathbb{R}^{n}$. Then

$$
\begin{aligned}
u(y) & =\frac{1}{\left|y-x_{0}\right|^{n-2}} \varphi\left(\frac{y-x_{0}}{\left|y-x_{0}\right|^{2}}+x_{0}\right), \\
& =\left(\frac{\varepsilon}{\left(\varepsilon^{2}-\left|x_{0}-x_{1}\right|^{2}\right)\left|y-x_{0}\right|^{2}-2\left(y-x_{0}\right)\left(x-x_{0}\right)-1}\right)^{(n-2) / 2} .
\end{aligned}
$$

We hereby complete the proof of Theorem 1.2

\section{Acknowledgements}

S. Tang and L. Wang are supported by the China Scholarship Council for their study/research at the University of Oklahoma. S. Tang and L. Wang would like to thank Department of Mathematics at the University of Oklahoma for its hospitality, where this work has been done. The work of S. Tang is supported by the National Natural Science Foundation of China (Grant No. 11801426) and Natural Science Basic Research Plan in Shaanxi Province of China (Program No. 2017JQ1022).

\section{REFERENCES}

[1] M. Chipot, I. Shafrir and M. Fila, On the solutions to some elliptic equations with nonlinear Neumann boundary conditions. Comm. Advances in Diff. Equs. 1 (1996), 91-110. 
[2] J.F. Escobar, Uniqueness theorems on conformal deformation of metric, Sobolev inequalities, and eigenvalue estimate. Comm. Pure Appl. Math. 43 (1990), 857-883.

[3] B. Gidas, W. Ni, L. Nirenberg, Symmetry and related properties via the maximum principle. Comm. Math. Phys. 68 (1979), 209-243.

[4] B. Gidas, W. Ni, L. Nirenberg, Symmetry of positive solutions of nonlinear elliptic equations in $\mathbb{R}^{n}$. Adv. in Math. Suppl. Stud., 7 A, Academic Press, New York, (1981), 369-402.

[5] B. $\mathrm{Hu}$, Nonexistence of a positive solution of the Laplace equation with a nonlinear boundary condition. Differ. Integral Equ., 7 (1994), 301-313.

[6] Y. Y. Li, L. Zhang, Liouville type theorems and Harnack type inequalities for semilinear elliptic equations. J. D'Anal. Math. 90 (2003), 27-87.

[7] Y. Y. Li, M. Zhu, Uniqueness theorems through the method of moving spheres. Duke Math. J. 80 (1995), 383-417.

[8] Y. Lou, M. Zhu, Classification of nonnegative solutions to some elliptic problems. Differ. Integral Equ. 12 (1999), 601-612.

[9] B. Ou, Positive harmonic functions on the upper half space satisfying a nonlinear boundary condition. Differ. Integral Equ. 9 (1996), 1157-1164.

Sufang Tang, School of Statistics, Xi'an University of Finance and Economics, Xi'an, ShaAnXi, 710100, P. R. China

E-mail address: sufangtang@163.com

Lei Wang, Academy of Mathematics and Systems Sciences, Chinese Academy of Sciences, Beijing 100190, P.R. China, and Department of Mathematics, The University of OKLahoma, Norman, OK 73019, USA

E-mail address: wanglei@amss.ac.cn

Meijun Zhu, Department of Mathematics, The University of Oklahoma, Norman, OK 73019, USA

E-mail address: mzhu@math.ou.edu 